\title{
TRANSFORMATION OF A CLASS OF NON-SELF-ADJOINT EIGENVALUE PROBLEMS
}

\author{
D. R. K. S. RAO AND K. N. MURTY
}

\begin{abstract}
A class of non-self-adjoint boundary value problems possessing countably many real eigenvalues can be made self-adjoint by means of a nonsingular transformation. A set of criteria for such problems to be self-adjoint is derived.
\end{abstract}

1. Introduction. Non-self-adjoint eigenvalue problems occur both in mathematical and physical problems. Solving non-self-adjoint eigenvalue problems is usually very difficult involving tedious calculations in complex analysis. The eigenvalues for a non-self-adjoint boundary value problem need not be real, may be finite or infinite and in some cases may not exist at all. But for a self-adjoint problem there are countably many real eigenvalues, and the eigenfunction expansion becomes simple and standard.

The fact that any scalar linear differential operator of second order can be made self-adjoint [2] on multiplication by a suitable factor suggests that certain boundary value problems involving matrix differential operators can be transformed by means of a nonsingular transformation into a self-adjoint boundary value problem. For such problems the theory of self-adjoint boundary value problems can be profitably applied instead of using the theory of non-self-adjoint boundary value problems.

$\$ 2$ deals with a set of criteria under which a given eigenvalue problem can be transformed into a self-adjoint eigenvalue problem, whereas $\$ 3$ analyzes the conditions deduced in $\$ 2$ on the constant nonsingular skew-Hermitian matrix $C$. Specific examples are given in each section.

2. Transformation of eigenvalue problems. In this section, we consider the general first order matrix differential equation

$$
L(y) \equiv P(t) y^{\prime}+Q(t) y=\lambda y, \quad(a<t<b),
$$

where $P(t) \in C^{2}, Q(t) \in C^{1}$ are square matrices of order $n, P(t)$ being nonsingular, $y$ is a column matrix with components $\left(y_{1}, y_{2}, \ldots, y_{n}\right), \lambda$ is a scalar parameter, and $(a, b)$ is a finite interval.

$A, B$ and $T$ stand for $(n \times n)$ matrix functions of $t . Y$ stands for a fundamental matrix of $y^{\prime}=A y, Z$ stands for a fundamental matrix of $y^{\prime}=-B^{*} y\left(B^{*}\right.$ is the transposed complex conjugate matrix of $B$ ).

\footnotetext{
Received by the editors July 11, 1979 and, in revised form, December 31, 1979.

AMS (MOS) subject classifications (1970). Primary 34A30.

Key words and phrases. Matrix differential equation, boundary value problems, fundamental matrix, elementary divisors.
} 
TheOREM 2.1. $T$ is a solution of $T^{\prime}=A T-T B$ if and only if $T=Y C Z^{*}$ where $C$ is a constant matrix.

Proof. It is easily verified that $Y C Z^{*}$ is a solution of $T^{\prime}=A T-T B$. Now to prove that every solution is of this form, let $T$ be a solution and $K$ be defined by $K=Y^{-1} T$. Then $Y^{\prime} K+Y K^{\prime}=A Y K-Y K B$ or $K^{* \prime}=-B^{*} K^{*}$. Since $Z$ is a fundamental matrix of $y^{\prime}=-B^{*} y$, it follows that there exists a constant matrix $C$ such that $K^{*}=Z C^{*}$ or $T=Y C Z^{*}$.

Theorem 2.2. Let $A=-\left(P^{-1} Q\right)$ and $B=\left(P^{*-1} Q^{*}-P^{*-1} P^{* \prime}\right)$. Then $W$ is a fundamental matrix of $y^{\prime}=B y$ if and only if $S=P^{-1} W^{*-1}$ is a fundamental matrix of $y^{\prime}=A y$.

Proof. The proof can be verified directly.

Definition 2.1. We shall say that the equation $L(y)=\lambda y, a<t<b$, can be transformed into a self-adjoint equation by the nonsingular $(n \times n)$ matrix-valued function $T$ if

$$
L_{1}(z)=T^{-1} L(T z)=\lambda z
$$

is a self-adjoint equation, and we shall say that the eigenvalue problem,

$$
L(y)=\lambda y, \quad M y(a)+N y(b)=0,
$$

can be transformed into a self-adjoint eigenvalue problem by $T$ if the problem

$$
L_{1}(z)=\lambda z, \quad M T(a) z(a)+N T(b) z(b)=0
$$

is self-adjoint.

Theorem 2.3. If $A, B$ are as in Theorem 2.2, then the general solution of $T^{\prime}=A T-T B$ is of the form $Y C^{*} Y^{*} P^{*}$, where $C$ is a constant matrix.

Proof. It is easily verified that $Y C^{*} Y^{*} P^{*}$ is a solution of $T^{\prime}=A T-T B$. Further, if $T$ is any solution of $T^{\prime}=A T-T B$, then $K=Y^{-1} T$ satisfies $K^{* \prime}=$ $-B^{*} K^{*}$. By Theorem 2.2, it follows that there exists a constant matrix $C$ such that $K^{*}=P Y C$ and hence $T=Y C^{*} Y^{*} P^{*}$.

THEOREM 2.4. The (differential) equation

$$
L(y) \equiv P(t) y^{\prime}+Q(t) y=\lambda y \quad(P \neq I), a<t<b,
$$

is transformed into a self-adjoint equation if and only if there exists a constant nonsingular skew-Hermitian matrix $C$ such that $Y C^{*} Y^{*} P^{*}$ is a positive definite Hermitian matrix.

Proof. Let the equation be transformed into a self-adjoint equation by means of a nonsingular matrix. Then

$$
L_{1}(z) \equiv T^{-1} L(T z)=\left(T^{-1} P T\right) z^{\prime}+\left(T^{-1} P T^{\prime}+T^{-1} Q T\right) z=\lambda z
$$

is self-adjoint if and only if

$$
\left(T^{-1} P T\right)^{*}=-\left(T^{-1} P T\right)
$$

and

$$
\left(T^{-1} P T\right)^{\prime}=\left(T^{-1} P T^{\prime}\right)+\left(T^{-1} Q T\right)-\left(T^{-1} P T^{\prime}\right)^{*}-\left(T^{-1} Q T\right)^{*}
$$


Equations (2.1) and (2.2) are equivalent to

$$
\begin{aligned}
& (P R)^{*}=-(P R), \\
& R^{\prime}=A R-R B,
\end{aligned}
$$

where $A=-\left(P^{-1} Q\right), B=\left(P^{*-1} Q^{*}-P^{*-1} P^{* \prime}\right)$ and $R=T T^{*}$. Therefore Theorem 2.3 implies that $R=Y C^{*} Y^{*} P^{*}$ for some constant matrix $C\left(2.1^{\prime}\right)$ shows that $C$ is skew-Hermitian and, since $T$ is nonsingular, $R=T T^{*}=Y C^{*} Y^{*} P^{*}$ is a positive definite Hermitian matrix.

Conversely, if there exists a constant nonsingular skew-Hermitian matrix $C$ such that $R=Y C^{*} Y^{*} P^{*}$ is a positive definite Hermitian matrix, then defining $T$ by $R=T T^{*}$ it is evident that $L_{1}$ is self-adjoint.

Here it is interesting to note from (2.1) that if $P$ is an identity matrix there will not exist such a nonsingular matrix $T$ transforming $L(y)$ into self-adjoint form.

TheOREM 2.5. Let $Y$ be a fundamental matrix of $y^{\prime}=A y, A=-\left(P^{-1} Q\right)$ and let $(M: N)$ be an $(n \times 2 n)$ matrix of rank $n$. The eigenvalue problem

$$
L(y) \equiv P(t) y^{\prime}+Q(t) y=\lambda y, \quad M y(a)+N y(b)=0
$$

can be transformed into a self-adjoint eigenvalue problem by a nonsingular matrix $T$ if and only if there exists a constant nonsingular skew-Hermitian matrix $C$ such that $Y C^{*} Y^{*} P^{*}$ is a positive definite Hermitian matrix and

$$
M Y(a) C Y^{*}(a) M^{*}=N Y(b) C Y^{*}(b) N^{*} .
$$

Proof. The transformed problem is

$$
L_{1}(z) \equiv P_{1} z^{\prime}+P_{2} z=\lambda z, \quad M_{1} z(a)+N_{1} z(b)=0,
$$

where $P_{1}=\left(T^{-1} P T\right), P_{2}=\left(T^{-1} P T^{\prime}+T^{-1} Q T\right), M_{1}=M T(a), N_{1}=N T(b)$. It is well known [1, p. 291] that (2.4) is self-adjoint if and only if $L_{1}(z)=\lambda z$ is self-adjoint (so that from Theorem 2.4, we have $Y C^{*} Y^{*} P^{*}$ is a positive definite Hermitian matrix), and $M_{1} P_{1}^{-1}(a) M_{1}^{*}=N_{1} P_{1}^{-1}(b) N_{1}^{*}$. The equalities $T T^{*}=$ $Y C^{*} Y^{*} P^{*}, M_{1} P_{1}^{-1}(a) M_{1}^{*}=N_{1} P_{1}^{-1}(b) N_{1}^{*}$ imply that

$$
M Y(a) C Y^{*}(a) M^{*}=N Y(b) C Y^{*}(b) N^{*} \text {. }
$$

Conversely, if there exists a constant nonsingular skew-Hermitian matrix $C$ such that $Y C^{*} Y^{*} P^{*}$ is a positive definite Hermitian matrix and $M Y(a) C Y^{*}(a) M^{*}=$ $N Y(b) C Y^{*}(b) N^{*}$, then defining $T$ by $T T^{*}=Y C^{*} Y^{*} P^{*}$, it is easy to verify that $T$ transforms the problem (2.3) into a self-adjoint problem.

We use the following notation.

$$
\operatorname{Diag}\left(a_{11}, a_{22}\right)=\left(\begin{array}{cc}
a_{11} & 0 \\
0 & a_{22}
\end{array}\right), \quad \operatorname{Off} \operatorname{Diag}\left(a_{12}, a_{21}\right)=\left(\begin{array}{cc}
0 & a_{12} \\
a_{21} & 0
\end{array}\right) .
$$

Then any $(2 \times 2)$ matrix $A$ can be written in the form

$$
A=\operatorname{Diag}\left(a_{11}, a_{22}\right)+\operatorname{Off} \operatorname{Diag}\left(a_{12}, a_{21}\right) \text {. }
$$

To illustrate the theorems of this section we consider the problem

$$
\begin{gathered}
L(y) \equiv \operatorname{Off} \operatorname{Diag}\left(1,-\frac{1}{4}\right) y^{\prime}+\operatorname{Diag}(-1,1) y=\lambda y, \\
\operatorname{Diag}(1,1) y(a)+\operatorname{Off} \operatorname{Diag}(1,-1) y(b)=0 .
\end{gathered}
$$


A fundamental matrix for $y^{\prime}=\operatorname{Off} \operatorname{Diag}(-4,-1) y$ is given by $Y(t)=$ $\operatorname{Diag}\left(2 e^{2 t}, e^{-2 t}\right)+\operatorname{Off} \operatorname{Diag}\left(2 e^{-2 t},-e^{2 t}\right)$. Also $C=\operatorname{Off} \operatorname{Diag}(-1,1)$ is a constant nonsingular skew-Hermitian matrix satisfying the self-adjointness condition (2.5). $T=\operatorname{Diag}(2,1)$ satisfies the condition $T T^{*}=Y C^{*} Y^{*} P^{*}$.

The transformed eigenvalue problem

$$
\begin{gathered}
L_{1}(z)=\operatorname{Off} \operatorname{Diag}\left(\frac{1}{2},-\frac{1}{2}\right) z^{\prime}+\operatorname{Diag}(-1,1) z=\lambda z, \\
\operatorname{Diag}(2,1) z(a)+\operatorname{Off} \operatorname{Diag}(1,-2) z(b)=0
\end{gathered}
$$

is a self-adjoint problem.

It may be noted that the problems (2.3) and (2.4) have the same infinite number of real eigenvalues (since $T$ is a nonsingular matrix). The problem (2.3) does not satisfy the self-adjointness condition but still possesses countably many real eigenvalues.

3. Classification of the matrix $C$. In this section we give the general form of a constant nonsingular skew-Hermitian matrix $C$ for which (i) $Y C^{*} Y^{*} P^{*}$ is a Hermitian matrix (Corollary 3.2) and derive a condition under which $C$ can be chosen to satisfy (ii) $M Y(a) C Y^{*}(a) M^{*}=N Y(b) C Y^{*}(b) N^{*}$ (Theorem 3.2). From (i) it follows that $Y C^{*} Y^{*} P^{*}=P Y C Y^{*}$, which can be put in the form

$$
G C=-C G^{*}
$$

where $G=\left(Y^{-1} P Y\right)$ is a known matrix of order $n$. First we write down the elementary divisors of $G$ in the field of complex numbers.

$$
(G):\left(\lambda-\lambda_{1}\right)^{\left(p_{1}\right)},\left(\lambda-\lambda_{2}\right)^{\left(p_{2}\right)}, \ldots,\left(\lambda-\lambda_{u}\right)^{\left(p_{u}\right)} \quad\left(p_{1}+p_{2}+\cdots+p_{u}=n\right) \text {. }
$$

In accordance with these elementary divisors we reduce $G$ and $G^{*}$ to Jordan normal form [3, p. 215],

$$
G=U \tilde{G} U^{-1} \text { and } G^{*}=U^{*-1} \tilde{G}^{*} U^{*}
$$

where $U$ is a nonsingular matrix and $\tilde{G}$ and $\tilde{G}^{*}$ are Jordan matrices:

$$
\begin{aligned}
\tilde{G}(t) & =\left\{\lambda_{1} I_{\left(p_{1}\right)}+H_{\left(p_{1}\right)}, \lambda_{2} I_{\left(p_{2}\right)}+H_{\left(p_{2}\right)}, \ldots, \lambda_{u} I_{\left(p_{u}\right)}+H_{\left(p_{u}\right)}\right\}, \\
\tilde{G}^{*}(t) & =\left\{\bar{\lambda}_{1} I_{\left(p_{1}\right)}+H_{\left(p_{1}\right)}, \bar{\lambda}_{2} I_{\left(p_{2}\right)}+H_{\left(p_{2}\right)}, \ldots, \bar{\lambda}_{u} I_{\left(p_{u}\right)}+H_{\left(p_{u}\right)}\right\} .
\end{aligned}
$$

Replacing $G$ and $G^{*}$ by their expressions given in (3.2), we get

$$
\tilde{G} \tilde{C}=-\tilde{C} \tilde{G}^{*}
$$

where $\tilde{C}=U^{-1} C U^{*-1}$.

We now partition $\tilde{\tilde{C}}$ into $u^{2}$ blocks corresponding to the quasidiagonal form of the matrices $\tilde{G}$ and $\tilde{G}^{*}$.

$$
\tilde{C}=\left(C_{\alpha \beta}\right) \quad(\alpha=1,2, \ldots, u ; \beta=1,2, \ldots, u),
$$

where $C_{\alpha \beta}$ is of order $\left(P_{\alpha} \times p_{\beta}\right), p_{r}$ being the multiplicity of $\lambda_{r}$.

Using the rule of multiplication of a partitioned matrix by a quasidiagonal one and carrying out multiplication of (3.4), this equation splits up into $u^{2}$ matrix equations

$$
\begin{aligned}
& {\left[\lambda_{\alpha} I_{\left(p_{\alpha}\right)}+H_{\left(p_{\alpha}\right)}\right] C_{\alpha \beta}=-C_{\alpha \beta}\left[\bar{\lambda}_{\beta} I_{\left(p_{\beta}\right)}+\right.}\left.H_{\left(p_{\beta}\right)}\right] \\
&(\alpha=1,2, \ldots, u ; \beta=1,2, \ldots, u),
\end{aligned}
$$


which we rewrite as

$$
\left(\lambda_{\alpha}+\bar{\lambda}_{\beta}\right) C_{\alpha \beta}=-\left[H_{\left(p_{\alpha}\right)} C_{\alpha \beta}+C_{\alpha \beta} H_{\left(p_{\beta}\right)}\right] .
$$

Let us take one of the equations (3.5). Two cases arise [3].

(i) If $\lambda_{\alpha} \neq-\bar{\lambda}_{\underline{\beta}}$, then $C_{\alpha \beta}=0$.

(ii) If $\lambda_{\alpha}=-\lambda_{\beta}$, then an arbitrary regular upper triangular matrix $C_{\alpha \beta}$ satisfies the equation. For the structure of $C_{\alpha \beta}$ see [3, p. 218].

Theorem 3.1. Let $C_{1}, C_{2}, \ldots, C_{N}$ be $N$ linearly independent solutions of the matrix equation (3.1). Then any solution of the matrix equation (3.1) in $C$ is given by

$$
C=\sum_{j=1}^{N} d_{j} C_{j}
$$

where $d_{1}, d_{2}, \ldots, d_{N}$ are $N$ parameters $\left(C_{j}\right.$ is obtained from $C$ by giving $d_{j}$ the value 1 and the remaining parameters the value $0 ; j=1,2, \ldots, N)$, and $N$ is determined by $N=\sum_{\alpha, \beta=1}^{u} \delta_{\alpha \beta}$ where $\delta_{\alpha \beta}=\min \left(p_{\alpha}, p_{\beta}\right)$.

Proof. The proof is similar to the proof of Theorem 1 in $[3$, p. 219].

Corollary 3.1. A necessary and sufficient condition for $C$ to represent a constant matrix is that the $d_{j}$ 's in (3.6) are constant.

COROLlARY 3.2. The general form of $C$ to represent a constant nonsingular skew-Hermitian matrix is given by $C=i\left(C_{\alpha \beta}\right), \alpha, \beta=1,2, \ldots, u$, where $C_{\alpha \beta}$ is a scalar matrix with real diagonal elements $d_{\alpha \beta}$ which is 0 if $p_{\alpha} \neq p_{\beta}$.

Substituting the general form of a constant nonsingular skew-Hermitian matrix $C$ in the self-adjoint boundary condition and splitting $M Y(a)$ and $N Y(b)$ into $u^{2}$ blocks corresponding to the splitting of the matrix $C$ into $C_{\alpha \beta}(\alpha=1,2, \ldots, u$; $\beta=1,2, \ldots, u)$, we get the system of equations $A x=0$, where

$$
\begin{aligned}
A & =a_{i j}^{\lambda \mu}=r_{\lambda i} \bar{r}_{\mu j}-s_{\lambda i} \bar{s}_{\mu j} \quad(i, j=1,2, \ldots, u ; \lambda, \mu=1,2, \ldots, u), \\
x^{T} & =\left(d_{11}, d_{12}, \ldots, d_{1 u}, d_{21}, \ldots, d_{2 u}, \ldots, d_{u 1}, \ldots, d_{u u}\right), \\
M Y(a) & =\left(r_{\alpha \beta}\right) \quad \text { and } N Y(b)=\left(s_{\alpha \beta}\right) \quad(\alpha=1,2, \ldots, u ; \beta=1,2, \ldots, u) .
\end{aligned}
$$

Hereafter we use the following notation:

$$
x=\left(d_{11}, d_{12}, \ldots, d_{1 u}, d_{21}, \ldots, d_{2 u}, \ldots, d_{u 1}, \ldots, d_{u u}\right)^{T} \text { and } X=\left(d_{\alpha \beta}\right)
$$

where $d_{\alpha \beta}$ are real $(\alpha=1,2, \ldots, u ; \beta=1,2, \ldots, u)$.

THEOREM 3.2. Let $A$ be $a\left(u^{2} \times u^{2}\right)$ matrix over the field of complex numbers and $|A|=0$. Every nontrivial solution of $A x=0$ has the property $|X| \neq 0$ if and only if there exists $x_{0} \in E^{u^{2}}$ such that $\left|X_{0}\right| \neq 0$ and row space of $A$ is equal to the orthogonal complement of $S=\left\{\alpha \bar{x}_{0} / \alpha \in \mathbf{C}\right\}$ in $E^{u^{2}}$.

Proof. Suppose every nontrivial solution of $A x=0$ has the property $|X| \neq 0$. Then the solution space of $A x=0$ is one dimensional because if $x_{1}$ and $x_{2}$ are linearly independent solutions with $\left|X_{1}\right| \neq 0$ and $\left|X_{2}\right| \neq 0$ then there exists an $\alpha \in \mathbf{C}$ such that $\left|X_{1}+\alpha X_{2}\right|=0$ and $\left(x_{1}+\alpha x_{2}\right)$ is a nontrivial solution. Let $x_{0}$ be 
the basis of the solution space of $A x=0$. Since $\rho(A)=u^{2}-1$, clearly the row space of $A$ is equal to the orthogonal complement of $S$ in $E^{u^{2}}$.

Conversely, suppose there exists a vector $x_{0}$ such that $\left|X_{0}\right| \neq 0$ and the row space of $A$ is the orthogonal complement of $S$. Then $x \neq 0, A x=0 \Rightarrow x=\alpha x_{0}$ for some scalar $\alpha \Rightarrow|X|=(\alpha)^{u}\left|X_{0}\right| \neq 0$.

To illustrate the theorems of this section, we consider the non-self-adjoint eigenvalue problem

$$
\begin{gathered}
L(y)=\operatorname{Off} \operatorname{Diag}\left(-e^{-2 t}, 4 e^{-2 t}\right) y^{\prime}+\operatorname{Off} \operatorname{Diag}\left(e^{-2 t},-4 e^{-2 t}\right) y=0, \\
{[\operatorname{Diag}(1,0)+\operatorname{Off} \operatorname{Diag}(1,0)] y(a)+[\operatorname{Diag}(0,1)+\operatorname{Off} \operatorname{Diag}(0,1)] y(b)=0 .}
\end{gathered}
$$

A fundamental matrix for $y^{\prime}=\operatorname{Diag}(1,1)$ is given by $Y(t)=\operatorname{Diag}\left(e^{t}, e^{t}\right)$. Therefore $G=Y^{-1} P Y=\operatorname{Off} \operatorname{Diag}\left(-e^{-2 t}, 4 e^{-2 t}\right)$. The elementary divisors of $G$ and $G^{*}$ are

$$
(G):\left(\lambda-2 i e^{-2 t}\right),\left(\lambda+2 i e^{-2 t}\right), \quad\left(G^{*}\right):\left(\lambda+2 i e^{-2 t}\right),\left(\lambda-2 i e^{-2 t}\right) \text {. }
$$

In accordance with these elementary divisors we reduce $G$ and $G^{*}$ into Jordan normal form

$$
G=U \tilde{G} U^{-1}, \quad G^{*}=U^{*-1} \tilde{G}^{*} U^{*},
$$

where $U=\operatorname{Diag}(i, 1)+\operatorname{Off} \operatorname{Diag}(1 / 2 i, 2)$. Let

$$
C=\operatorname{Diag}\left(C_{11}, C_{22}\right)+\operatorname{Off} \operatorname{Diag}\left(C_{12}, C_{21}\right)
$$

Then from condition (3.4) we get

$$
\begin{aligned}
{\left[\operatorname { D i a g } \left(2 i e^{-2 t},\right.\right.} & \left.\left.-2 i e^{-2 t}\right)\right]\left[\operatorname{Diag}\left(C_{11}, C_{22}\right)+\operatorname{Off} \operatorname{Diag}\left(C_{12}, C_{21}\right)\right] \\
& =-\left[\operatorname{Diag}\left(C_{11}, C_{22}\right)+\operatorname{Off} \operatorname{Diag}\left(C_{12}, C_{21}\right)\right]\left[\operatorname{Diag}\left(-2 i e^{-2 t}, 2 i e^{-2 t}\right)\right] .
\end{aligned}
$$

Solving for $C$, we get $C_{12}=C_{21}=0$. Since $C$ has to be a constant nonsingular skew-Hermitian matrix, it follows that the general form of $C$ is $\operatorname{Diag}\left(\mathrm{id}_{11}, \mathrm{id}_{22}\right)$. Substituting this in the self-adjointness condition and expanding we get the system of equations $A x=0$. There is a nontrivial solution $d_{11}, d_{22} \neq 0$ satisfying the condition $|X| \neq 0$.

The authors are grateful for the criticisms and suggestions of the referee which resulted in the improvement of this paper.

\section{REFERENCES}

1. E. A. Coddington and N. Levinson, Theory of ordinary differential equations, McGraw-Hill, New York, 1955.

2. E. L. Ince, Ordinary differential equations, Longmans, Gree and Co., Ltd., London, 1927.

3. F. R. Gantmacher, The theory of matrices. I, Chelsea, New York, 1959.

Department of ApPlied Mathematics, ANDhra University, Waltair 530003, India 